\title{
Performance Analysis of MAC Layer using Multicast Routing over Resource Constrained Ad Hoc Networks
}

\author{
Amit Chopra \\ PhD Research Scholar, \\ CSE Deptt.,MMEC, \\ M. M. University, Ambala, India
}

\author{
Rajneesh Kumar, PhD \\ Professor \\ CSE Deptt., MMEC, \\ M. M. University, Ambala, India
}

\begin{abstract}
Group communication over multicast ad hoc network suffers from insufficient utilization of limited resources, i.e. shared channel, battery, data processing capabilities and storage space, etc. Multicast routing protocol should be able to manage all these resources because their consumption depends upon different factors, i.e. Unicast/Multicast network operations, dynamic topology due to mobility, control overhead due to scalability, packet loss and retransmission due to collision and congestion etc. All these factors may cause unnecessary network load, delay and unfair resource utilization. However, multicast ad hoc routing protocols are more efficient than Unicast routing protocols, but they also suffer from performance degradation factors discussed above. Researchers have developed various layer wise solutions for resource optimization. In this paper, we will explore the different schemes for fair utilization of network resources and also perform a simulation based analysis to investigate the impact of MAC layer over the performance of multicast routing protocols and network resources.
\end{abstract}

\section{Keywords}

Multicast, Ad Hoc, Wireless, QoS, Resource Management, Bandwidth, Energy Efficient

\section{INTRODUCTION}

\subsection{Multicast Ad Hoc Networks}

Fair utilization of network resources is a major issue for multicast ad hoc networks where network operations are simultaneously performed by the different layers. Multicast protocol dependency over multiple layers may degrade the performance of the entire network, if any intermediate layer exhausts maximum resources for data processing. So it is essential to optimize the operation of each layer for efficient utilization. Following are the operations supported by different layers: [1]

Table 1 Layer wise network operation(s)

\begin{tabular}{|c|c|}
\hline Layer(s) & Operation(s) \\
\hline Application Layer & $\begin{array}{c}\text { Scalable Encoding, Error Correction, } \\
\text { Adaptive Joint Source/Channel Coding, } \\
\text { Network Coding for Relay Networking }\end{array}$ \\
\hline Transport Layer & Error Correction \\
\hline Network Layer & Network Coding for Relay Networking \\
\hline MAC Layer & Unicast packets processing, Polling, \\
\hline
\end{tabular}

\begin{tabular}{|c|c|}
\hline & $\begin{array}{c}\text { Error Detection/Correction, } \\
\text { Packet Retransmission }\end{array}$ \\
\hline Physical Layer & Signal Processing, \\
& Space Time Coding, \\
& Resource Allocation \\
\hline
\end{tabular}

Operations of all these layers can be optimized for efficient utilization of limited resources. In section-II, we will explore the contribution of different researchers in relevant areas.

\subsection{Resource Categorization}

Table 2 below shows the various categories of resources and their utilization in different network operations. Each resource has some sort of constraints and there is need to develop an optimal solution for fair utilization of limited resources.

Table 2 Resource Categorization1-2

\begin{tabular}{|c|c|c|c|}
\hline $\begin{array}{c}\text { Available } \\
\text { Resource(s) }\end{array}$ & $\begin{array}{c}\text { Resource } \\
\text { Consumption }\end{array}$ & Limitation(s) & Requirement(s) \\
\hline $\begin{array}{l}\text { Battery } \\
\text { Power }\end{array}$ & $\begin{array}{c}\text { Packet } \\
\text { Transmission, } \\
\text { Packet } \\
\text { Reception, Data } \\
\text { Processing }\end{array}$ & $\begin{array}{l}\text { Low battery } \\
\text { backup }\end{array}$ & $\begin{array}{c}\text { Energy } \\
\text { Efficiency }\end{array}$ \\
\hline $\begin{array}{c}\text { Shared } \\
\text { Channel }\end{array}$ & Communication & $\begin{array}{l}\text { Limited/Shared } \\
\text { bandwidth, } \\
\text { unfair channel } \\
\text { allocation, } \\
\text { Collision }\end{array}$ & $\begin{array}{c}\text { Fair Channel } \\
\text { Allocation, } \\
\text { Optimum } \\
\text { bandwidth } \\
\text { utilization }\end{array}$ \\
\hline $\begin{array}{c}\text { Central } \\
\text { Processing } \\
\text { Unit (CPU) } \\
\end{array}$ & $\begin{array}{c}\text { Data } \\
\text { Processing }\end{array}$ & $\begin{array}{c}\text { Low } \\
\text { Processing } \\
\text { Power } \\
\end{array}$ & $\begin{array}{c}\text { Fair Job } \\
\text { Scheduling }\end{array}$ \\
\hline $\begin{array}{l}\text { Node's } \\
\text { Memory }\end{array}$ & Data Storage & Low Capacity & $\begin{array}{c}\text { Memory } \\
\text { Optimization }\end{array}$ \\
\hline
\end{tabular}

\subsection{Design goals of Multicast Routing Protocols for Ad Hoc Networks}

To develop a multicast routing protocol, there is a need to focus on various constraints like of dynamic topology, limited power, shared bandwidth, network security and scalability. Ad hoc Multicast routing protocols should be robust, efficient and reliable. It should manage the control overhead, QoS, dependency over unicast protocol and limited resources. Fig.1 below shows the classification of multicast routing protocols: 


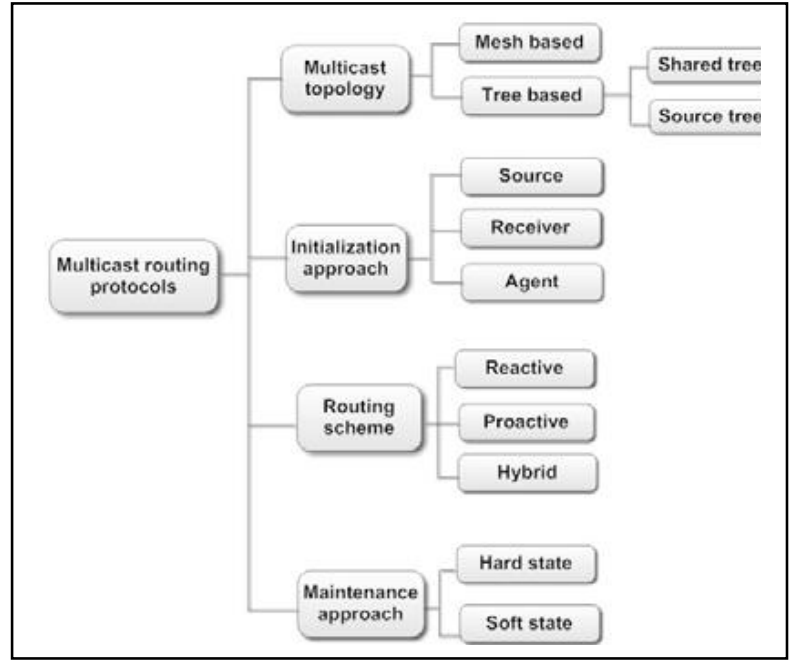

Fig .1 Classifications of multicast routing protocols [3]

\subsection{Classification of Multicast Routing Protocols for Ad Hoc Network}

1. Multicast Topology: Ad hoc multicast routing protocols can be classified into two types: tree based and mesh based etc. In case of tree based, single link is established between the source and destination pair whereas in mesh based approach, there may be more than one link between the source and destination pair. Tree based multicast protocols are most efficient, but mesh based multicast protocols are more robust. Tree based multicast protocols can have the following types:

a. Source-Tree-based: In which each source maintains a separated tree that contains the source node as the root of the tree and all receivers lies under this node.

b. Shared-Tree-based: In which one tree is established in the entire network which includes all sources and receivers and links are routed to a main node that is referred core node [2][3].

2. Routing Initialization Approach: Multicast group formation can be initiated by source as well as by receivers. Routing initialization can be classified into three approaches:
a. Source-initiated: Source node initiates the group formation
b. Receiver-initiated: Receivers initiate the group formation
c. Hybrid approach: It uses the combination of both approaches

3. Routing Scheme: Routing scheme is classified into three approaches:

a. Proactive approach: Nodes maintain the routing tables for network topology

b. Reactive approach: Routes are established as per requirements.

c. Hybrid approach: It uses the combination of both approaches [2][3].
4. Maintenance Approach: It is classified into two different approaches:

a. Soft State: Multicasting Tree is updated periodically using control packets

b. Hard State: It is similar to Soft State, except that it also uses a route repair method[2].

\subsection{MAC Layer design goals and issues}

MAC protocol should consider the various facts which can reduce its performance over ad hoc networks, i.e. Hidden and Exposed terminal issues, dynamic topology, fair channel allocation, scalability, link quality and cross layer communication, etc. MAC protocol should support: dynamic and distributed network environment, QoS, Data Synchronization and bandwidth management, etc. [2][23]

\subsection{MAC Layer Backoff Algorithms}

Shared channel can be simultaneously accessed by multiple nodes and it may result in collisions. In order to avoid the collision over the channel, MAC layer uses backoff algorithms to calculate the waiting period for the nodes. Following are few Backoff algorithms: [22][23]

Table 3 MAC Layer BAckoff Algorithms

\begin{tabular}{|c|c|c|c|c|}
\hline \multirow{2}{*}{$\begin{array}{c}\text { MAC } \\
\text { Layer } \\
\text { Backoff } \\
\text { Algorithms }\end{array}$} & \multicolumn{3}{|c|}{$\begin{array}{c}\text { Contention Window Size Estimation } \\
\text { (cont_win_s) }\end{array}$} & \multirow[b]{2}{*}{$\begin{array}{c}\text { Cons } \\
\text { t. } \\
\text { Valu } \\
\text { e }\end{array}$} \\
\hline & $\begin{array}{l}\text { Collision } \\
\text { Stage } \\
\text { (Ts }= \\
\text { Time_Slot) }\end{array}$ & $\begin{array}{c}\text { Normal } \\
\text { Stage }\end{array}$ & $\begin{array}{l}\text { Min_cont_ } \\
\text { win_s }\end{array}$ & \\
\hline $\begin{array}{c}\text { Binary } \\
\text { Exponential }\end{array}$ & $\begin{array}{c}\text { (Const } * \\
\text { cont_win_s }) \\
\text { Ts }\end{array}$ & 32 & 32 & 2 \\
\hline $\begin{array}{c}\text { Modified } \\
\text { Backoff }\end{array}$ & $\begin{array}{c}\text { Const } * \\
\text { cont_win_s }{ }^{*} \text { Ts }\end{array}$ & 32 & 32 & 1.5 \\
\hline $\begin{array}{c}\text { Multiplicati } \\
\text { ve Increase } \\
\text { Linear } \\
\text { Decrease }\end{array}$ & $\begin{array}{c}\text { Const } * \\
\text { cont_win_s } * \\
\text { Ts }\end{array}$ & $\begin{array}{c}\text { cont_wi } \\
\text { n_s-1 }\end{array}$ & $\begin{array}{c}\text { cont_win_s } \\
-1\end{array}$ & 1.5 \\
\hline $\begin{array}{c}\text { Double } \\
\text { Increment } \\
\text { Double } \\
\text { Decrement }\end{array}$ & $\begin{array}{c}\text { Const } * \\
\text { cont_win_s } \\
\text { Ts }\end{array}$ & $\begin{array}{c}1 / 2 \\
\text { (cont_w } \\
\text { in_s) }\end{array}$ & cont_win_s & 2 \\
\hline $\begin{array}{l}\text { Logarithmi } \\
\text { c Backoff }\end{array}$ & $\begin{array}{c}\log (\text { cont_win } \\
\text { _s }) \\
\text { cont_win_s } \\
\text { Ts }\end{array}$ & $\begin{array}{r}\text { con } \\
\text { Min_cc }\end{array}$ & $\begin{array}{l}\text { win_s }= \\
\text { tt_win_s=32 }\end{array}$ & - \\
\hline $\begin{array}{l}\text { Pessimistic } \\
\text { Linear- } \\
\text { Exponential } \\
\text { Backoff }\end{array}$ & $\begin{array}{c}\text { cont_win_s } * \\
\text { Const^1/ } \\
\text { Const } * \text { Ts }\end{array}$ & $\begin{array}{l}\text { cont_wi } \\
\text { n_s-Ts }\end{array}$ & cont_win_s & 2 \\
\hline $\begin{array}{l}\text { Exponential } \\
\text { Increase } \\
\text { Exponential } \\
\text { Decrease } \\
\text { back-off }\end{array}$ & $\begin{array}{c}\text { Get_min_val( } \\
\text { back_off_fact } \\
\text { or* } \\
\text { cont_win_s, } \\
\text { Max_cont_wi } \\
\text { n_s) }\end{array}$ & $\begin{array}{l}\text { Get_1 } \\
\text { /back } \\
\text { Min_ }\end{array}$ & $\begin{array}{l}\text { ax_val(cont_ } \\
\text { in_s } \\
\text { off_factor, } \\
\text { ont_win_s) }\end{array}$ & \\
\hline $\begin{array}{c}\text { Fibonacci } \\
\text { Backoff }\end{array}$ & $\begin{aligned} & \text { cont_win_s } \\
&= \text { cont_win_s- } \\
& 1\end{aligned}$ & $\begin{array}{l}\text { cont__ } \\
\text { win_- } \\
\mathrm{s}+1= \\
\mathrm{f}(\mathrm{n})\end{array}$ & & $\begin{array}{l}\mathrm{f}(\mathrm{n})= \\
\mathrm{f}(\mathrm{n}-1) \\
+\mathrm{f}(\mathrm{n}-2\end{array}$ \\
\hline $\begin{array}{l}\text { Default } \\
\text { Backoff } \\
\text { Method }\end{array}$ & $\begin{array}{l}\text { (cont_win_ } \\
\mathrm{s} \ll 1)+1\end{array}$ & $\begin{array}{l}\text { cont_wi } \\
=\text { =Min_cc }\end{array}$ & st_win_s=32 & \\
\hline
\end{tabular}


This article contains different sections i.e. Section-I introduce the basic requirements of resource management for multicast communication and Section-II describes related research work in relevant field. It provides brief overview of the various solutions developed for efficient utilization of resources. Section-III highlights the open issues related to resource management and Section-IV shows simulation results and analysis and section V concludes the outcome of the analysis.

\section{EXISTING SOLUTIONS FOR NETWORK RESOURCE MANAGEMENT OVER AD HOC NETWORKS}

Y. Sun et al. [6] proposed an enhanced bandwidth efficient multicast routing for MANET. It optimizes multicast tree construction phase as well as a tree maintenance phase to reduce bandwidth consumption. Simulation results show its performance in terms of less control overhead with optimal bandwidth utilization.

Ritesh K. Kalle et al. [7] proposed an energy efficient method for VoIP communication over IEEE-802.16m based networks by reducing overheads. During the data transmission phase, mobile station remains active and uses packet buffering. As per their analytical analysis, they categorized the power consumption in different modes i.e. idle mode, transmission mode and receiving mode and finally they examined the cycles in each mode. Simulation results show that power can be preserved in sleep mode by optimizing the MAC layer, by reducing overhead and delay etc.

Jiwen Guo et al. [8] proposed a secure minimum-energy multicast (SMEM) algorithm to ensure multicast communication. In order to improve the stability of trust mechanism, the new trust values (calculated by the Bayesian theorem in CR networks) are modified by the iterative control criterion. The trust mechanism aims at guaranteeing the security of network environment, in which the trust information is encrypted to ensure the credibility of trust values. Results show that the time complexity of SMEM algorithm is polynomial.

S.M. Lakshmi et al. [9] developed an energy efficient routing method for multicast communication. Instead of using routing table, nodes use unique multicast id to transmit the data to a specific group. On demand routing path is built by calculating the residual energy of intermediate multicast nodes and the distance between them. Simulation results show its performance in terms of minimum delay, extended network life time and enhanced Throughput.

Di Marco et al. [10] introduced the study of routing metrics and interaction of routing with MAC. Different concepts of cross layer interactions are introduced. MAC layer parameters as well as network performance both are affected by Contention levels. Some reliability constraints are enforced to increase the lifetime of the network and also used to balance the network load. Proposed solutions are compared with the routing approach based on backpressure mechanism and the results show the efficiency of proposed solution.

Farooq M.O et al. [11] presented a bandwidth estimationbased admission control and routing protocol, called BEAR for IEEE 802.15.4 - based networks. The purpose of BEAR is to support real-time multimedia flows in IEEE 802.15.4 based networks by satisfying their end-to-end bandwidth requirements. It combines a bandwidth estimation approach, admission control, and routing. It uses a measurement-based bandwidth estimation algorithm that combines nodes' transmission rate, and actual MAC layer overhead to estimate the available bandwidth. The available bandwidth information is used as input to the admission control protocol, and it supports algorithms to estimate a flow's contention, intra-flow contention, and additional MAC layer overhead with an increased data load inside a network. Available bandwidth information can be used as a routing metric to select forwarding path(s) that can offer better end-to-end available bandwidth. Proposed framework can be used to support realtime multimedia applications for IEEE 802.155.4 based networks.

Jackson.C.A et al. [12] proposed a hierarchical and heterogeneous multichannel ad hoc network. The channels employed by this network are non-overlapping, and each channel differs significantly in its characteristics, such as achievable data rate, communication range, and traffic load. Every terminal is connected with the frequency-agile radio, which can change its transmission rate and carrier frequency. These radios communicate using contention-based access and are permitted to utilize multiple channels. A subset of terminals forms a backbone network, which is equipped with a second radio tuned to a traffic channel employing schedulebased access. They considered various hierarchical and heterogeneous multichannel ad hoc network topologies and investigated the backbone networks that increase networklayer performance over scenarios in which too few or too many terminals are selected to form the backbone network.

Xueyuan Su et al. [13] focused on the bandwidth allocation problem and explored the network and MAC layer impact over the existing bandwidth allocation methods, i.e. Interference model, Rate allocation scheme and contention based algorithms. The study shows that cross layer solutions can be used to regulate the data flow rate across both layers.

Xiaoying Zhang et al. [14] developed an energy efficient, cooperative MAC Protocol, which utilizes Time and Space estimation based Backoff algorithms to manage the impact of channel interference. To transmit data, it regulates the MAC layer and reduces the energy consumption. Simulation results show its performance in terms of an enhanced network lifetime as with maximum energy conservation during network operations, as compared to MAC 802.11 $\mathrm{DCF} / \mathrm{Cooperative} \mathrm{MAC} \mathrm{protocol.}$

D. S. Patil et al. [15] introduced an energy conservative MAC layer protocol, called PowerMAC which utilizes the GPS location of each node and can deliver the data using minimum Hop count. It uses Ad hoc on demand distance vector and Location aided routing protocols with a value of network allocation vector. If intermediate nodes have a minimum vector value, then data can be forwarded to avoid the transmission delay/packet loss. Each successful transmission reduces the frequency of packet retransmission and thus results in energy conservation. Simulation results show its performance in terms of data delivery using minimum hops towards the destination, end-to-end delay and energy consumption as compared to MAC 802.11

Xin Zhao et al. [16] proposed a reliable multicast routing method which considers link quality, packet retransmission at the MAC layer and packet broadcasting, etc. For bandwidth efficiency, they constructed a multicast tree by reducing all these factors. Simulation results show its performance in terms of the improved Packet delivery ratio, Throughput and reduction in packet retransmission. 
S. Chettibi et al.17 proposed a routing solution based on reinforcement learning method to enhance the network lifetime. The route discovery process is initiated on the basis of remaining energy of each node. If the node fulfill the energy consumption constrains, only then it is eligible to forward the Route request packets. After each iteration, all nodes adjust their packet forwarding rules as per their remaining energy levels. Finally, it results in a controlled packet forwarding over the entire network, thus also results in the reduction of RREQ packet flooding. Simulation results show its performance in terms of extended network lifetime, energy conservation and optimal routing with minimum delay as compared to Probalistic/Time and Delay methods.

S. B Kulkarni et al. [18] proposed a resource aware cluster based routing scheme for multicast communication over ad hoc networks. Cluster head is selected on the basis of various resources, i.e. bandwidth, energy level and node connectivity, etc. After cluster head selection, group members become leaf nodes and multicast tree is constructed on the basis of shortest paths. Analytical studies show that proposed scheme supports resource aware routing.

Gyanappa A. Walikar et al. [19] developed an energy efficient multicast routing scheme which constructs a multicast tree using reliable members only. Reliability of members is calculated on the basis of node density and remaining energy level. During tree maintenance, routing paths can be selected or dropped on the basis of energy level of intermediate nodes. Simulation results show that its performance in terms of higher PDR with less delay as compared to AODV/AOMDV routing protocols.

Yan Sun et al. [20] enhanced an existing bandwidth management scheme by reducing routing overhead, called IBEMR. It constructs a shared multicast tree on the basis of the group joining requests/replies. To avoid the collision, it estimates the total control/data packets to be transmitted. A multicast session is established for transmission purpose to minimize the cost of tree reconstruction due unstable network topology. Simulation analysis show that the IBMER can optimize the tree construction phase, thus results in fair bandwidth utilization, whereas reduction of control overhead can preserve the energy, as compared to ABAM and BEMR

K. Rajkumar et al. [21] proposed a resource aware routing solution for multicast communication, called Efficient Geographic Multicast Protocol. This protocol subdivides the entire geographical locations into small logical regions, called virtual zones. Zone leader is selected on the basis of its distance from the source and it communicates with all member nodes. Zone leader is responsible for data transmission to group members, thus reduces the unnecessarily broadcast over the entire network. Reliable packet transmission to various geographical locations is supported by EGMP as compared to Scalable Position Based Multicast Routing Protocol. Simulation results show its performance in terms of less packet retransmission, higher packet delivery ratio and effective resource management etc.

\section{PROBLEM FORMULATION}

\subsection{Multicast Routing Constraints}

MANET based applications are widely used in the remote areas where rapid deployment and dynamic reconfiguration is necessary and wired network support is unavailable. These include military battlefields, emergency search, rescue sites, classrooms and conventions, where communication can be initialized using audio/video streams with the help of wireless nodes. In case of voice communication over MANETs, we transmit audio stream having a high data rate and it reduces the voice quality due to the usage of low-power wireless links. Transmission of concurrent voice streams can reduce the efficiency of the entire network. Voice stream multicast can play an important role in case of emergency situations. In case of video communication over MANETs, it requires efficient bandwidth and real time support, but due to the limited resources and error prone network environment, it becomes challenging to manage the quality of video transmission as well as the network bandwidth.

Multicast routing plays a significant role in MANETs and multicasting can improve the efficiency of the wireless links, but it is difficult and challenging to design an effective and reliable multicast routing protocol for MANETs. In recent years, various multicast routing protocols have been proposed for MANETs to support different services. Multicast communication over ad hoc networks has its own limitations, but it also suffers from common issues, i.e. dynamic topology, performance of routing protocol, inefficient resource management, security, scalability, QoS, QoE etc. If we use the multicasting over the MANETs then there is need to explore and sort out these issues for the multicast mobile ad hoc networks [2].

Quality of communication is affected by noise/interference and channel fading. Multicast routing protocol must be able to regulate the flow of control packets over a network, in order to maintain the channel capacity4. There may be packet drop due to the dynamic change in the network topology that can reduce the network performance. So multicast routing should be able to operate in different situations such as mobile environment, heavy traffic load, scalability in order to achieve high packet delivery [2]

\subsection{Resource management issues}

Efficient resource utilization is a critical issue for Multicast Ad hoc networks, which deals with the consumption of various network resources discussed below:

a. Node Energy: Every node has limited battery life and it is very difficult to manage the energy level of individual node because each node may participate in different network operations and its energy level is reduced as per the performed task [2][5].

b. Shared Channel Bandwidth allocation: Ad hoc networks utilize shared channel and fair channel allocation becomes a major issue [8].

c. Node Level Buffer management: Due to packet loss/link loss, retransmission phase is executed by routing protocol, thus may result in a buffer overflow, network congestion/contention etc. [2]

\subsection{Resource utilization dependencies}

a. Network size/topology: Due to the dynamic network topology and network size, route discovery and route maintenance phase are performed by protocols and thus results in degradation of network performance.

b. Node density/mobility: Node Mobility and scalability can degrade the QoS of the entire network and it is difficult to maintain network performance under these constraints [1].

c. Traffic type/load: Real time applications consume network resources at large scale. In insufficient 
bandwidth allocation may result in delay and can degrade the quality of experience of end users.

\subsection{Behavioral Impact of different layers over network resource consumption}

a. Multicast Routing Layer: Routing layer performs different network operations such as route discovery, route maintenance and session management, etc. Routing layer operations may suffer from the dynamic behavior of the ad hoc networks and thus may result in the link errors and frequent update of routing information. Due to excessive route maintenance, node's life time can be exhausted [2].

b. MAC Layer: It communicates with two different layers, i.e. routing layer and physical layer. It also manages contention over wireless channel2. MAC protocols directly affect the channel allocation, bandwidth utilization, contention and packet collision, etc. Collision at large scale can produce the delay and contention over network and QoS may be degraded. If MAC protocols fail to manage fair channel allocation, it can also degrade the performance of ad hoc networks in a multi user environment.

\section{SIMULATION ANALYSIS AND RESULTS}

Table 4 Simulation Configuration

\begin{tabular}{|c|c|}
\hline Simulation Parameters & Parameter Values \\
\hline Multicast Routing Protocol(s) & MAODV, PUMA, MZRP \\
\hline Terrain & $1200 x 1200$ \\
\hline Node Density & 30 \\
\hline MAC Protocol & MAC 802.11 \\
\hline Backoff Algorithms & As per Section-I \\
\hline Traffic Type & CBR \\
\hline Packet Size & 512 \\
\hline Sampling Interval & 0.1 seconds \\
\hline Simulation Time & 10 seconds \\
\hline Network Simulator & NS-2.35 \\
\hline
\end{tabular}

\subsection{Performance Analysis}

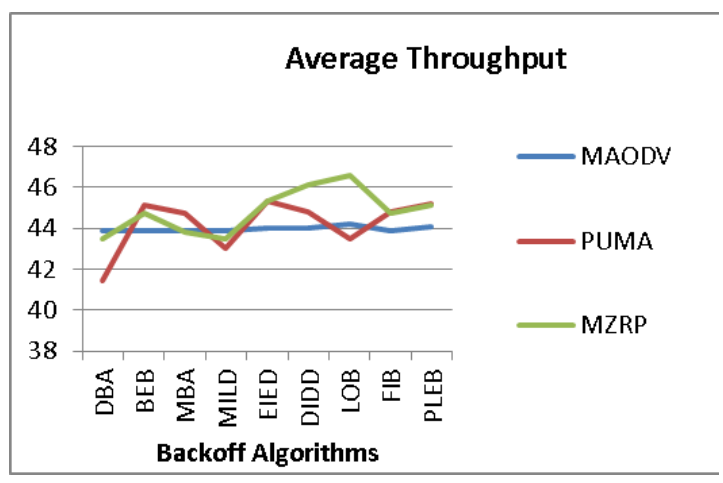

Fig .2 Average Throughput

The graphs below show Throughput, PDR, Routing Load, End-to-End Delay and number of Collisions using different routing protocols w.r.t. backoff algorithms. We can observe the impact of Backoff algorithms over the performance of the multicast routing protocols.

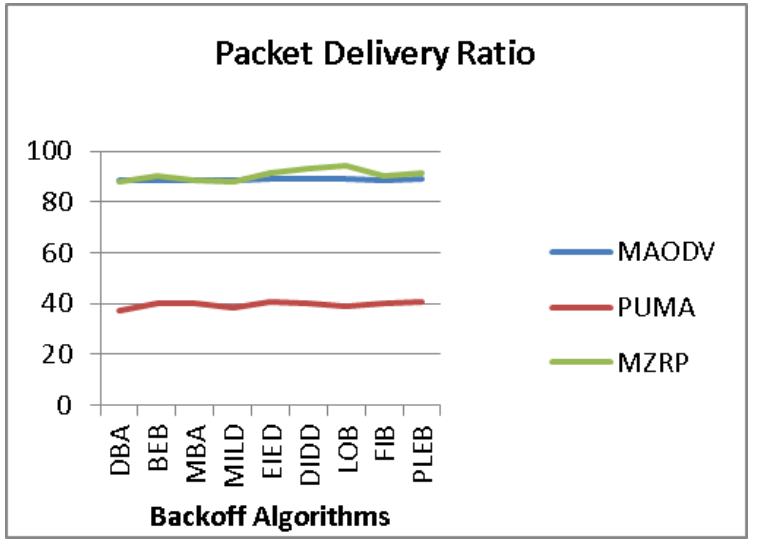

Fig .3 Packet Delivery Ratio

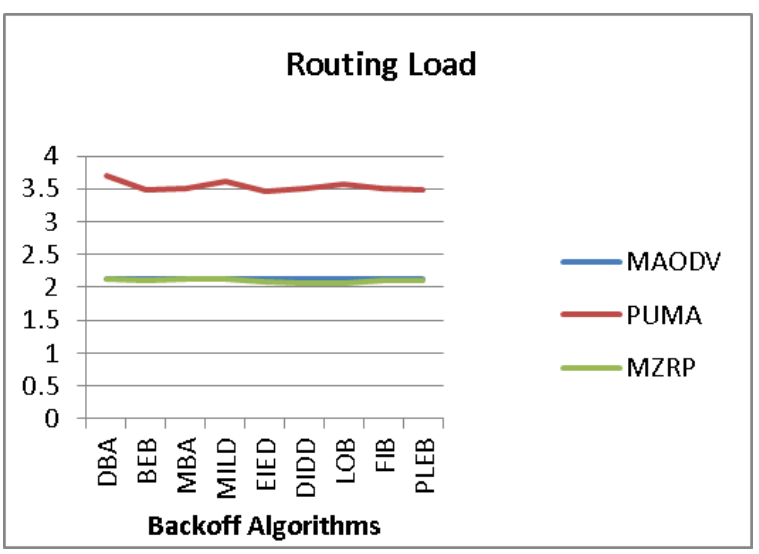

Fig .4 Routing Load

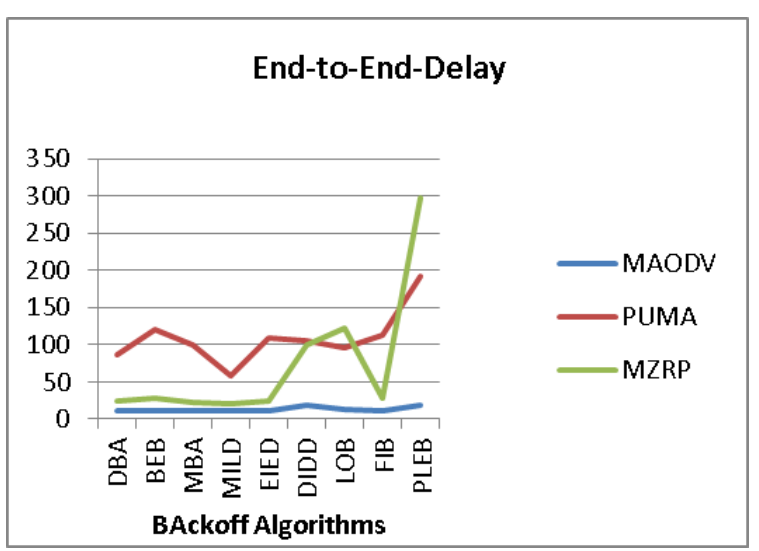

Fig .5 End-to-End Delay

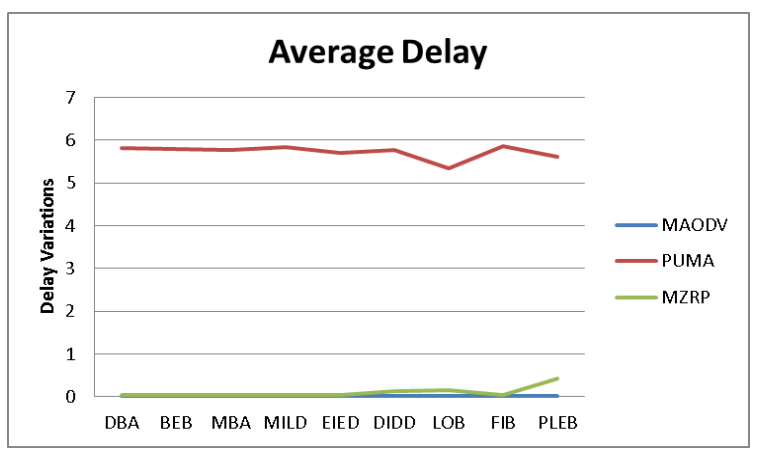

Fig .6 Average Delay 


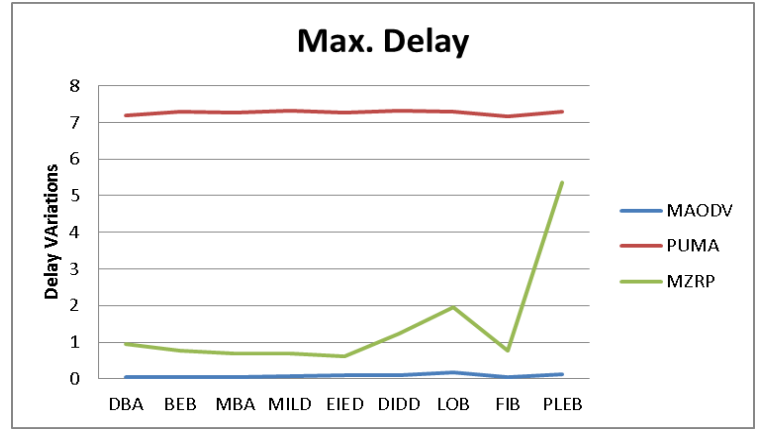

Fig .7 Max. Delay

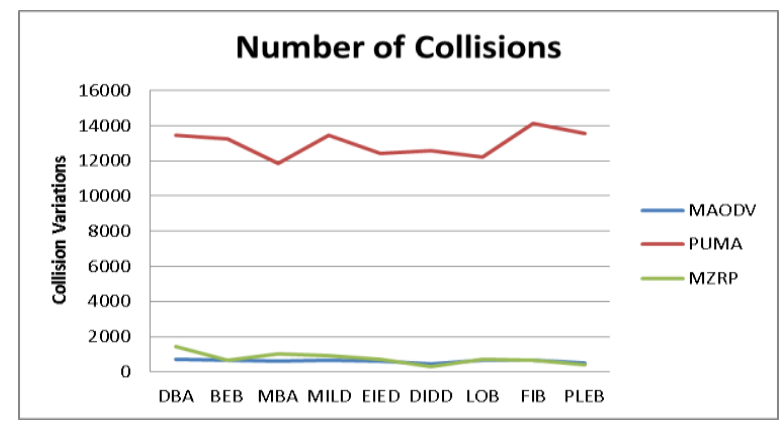

Fig .8 Number of Collisions

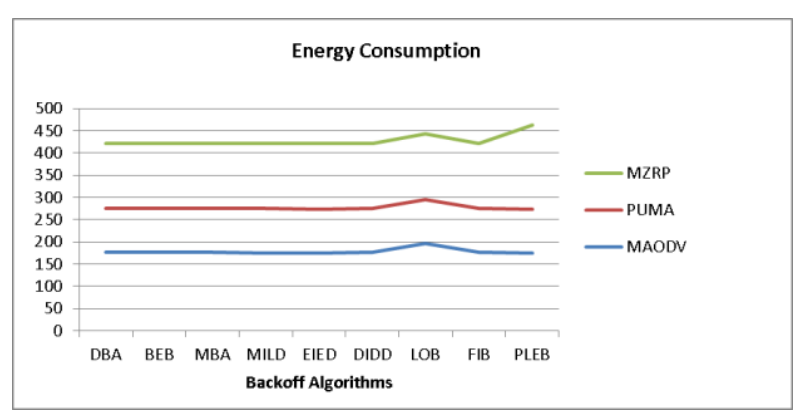

Fig .9 Energy Consumption

\subsection{Analysis of Node Level Energy Consumption}

Following graphs show energy consumption using routing protocols MAODV, PUMA and MZRP with various Backoff algorithms.

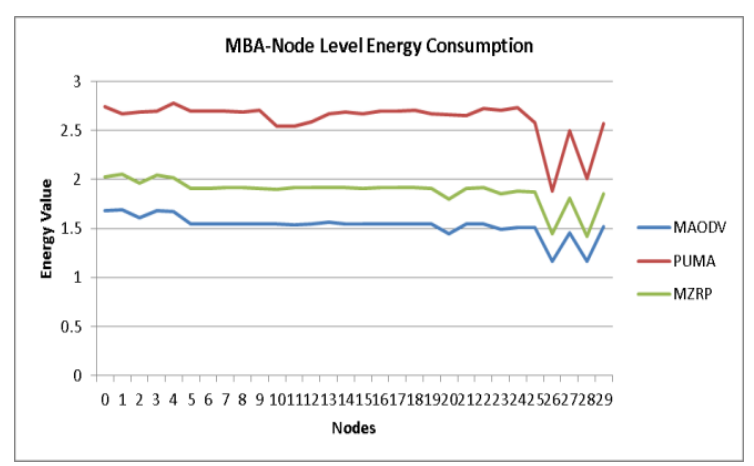

Fig .10 DBA-Node Level Energy Consumption

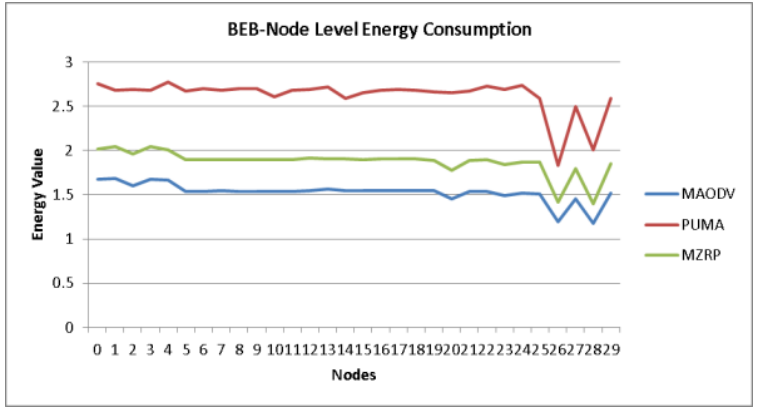

Fig .11 BEB- Node Level Energy Consumption

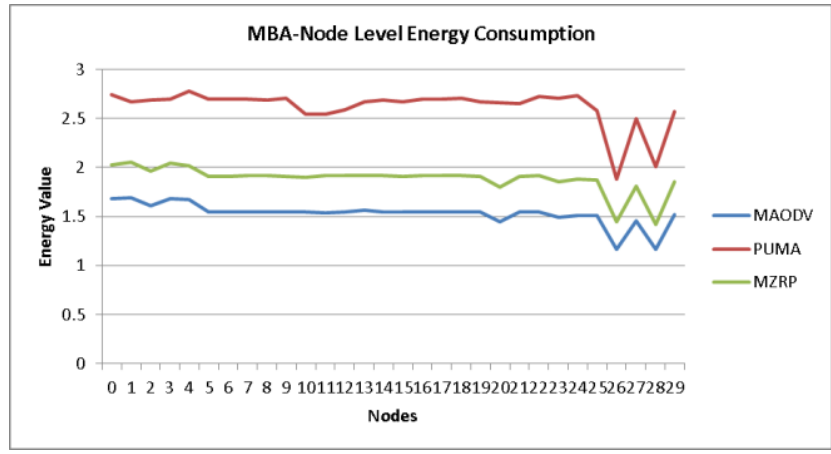

Fig .12 MBA- Node Level Energy Consumption

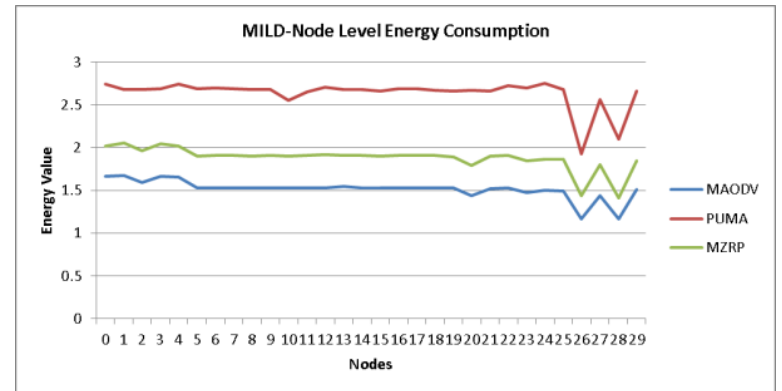

Fig .13 MILD- Node Level Energy Consumption

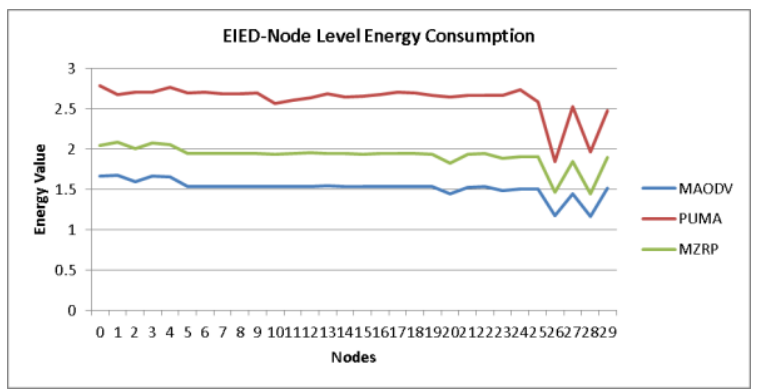

Fig .14 EIED- Node Level Energy Consumption

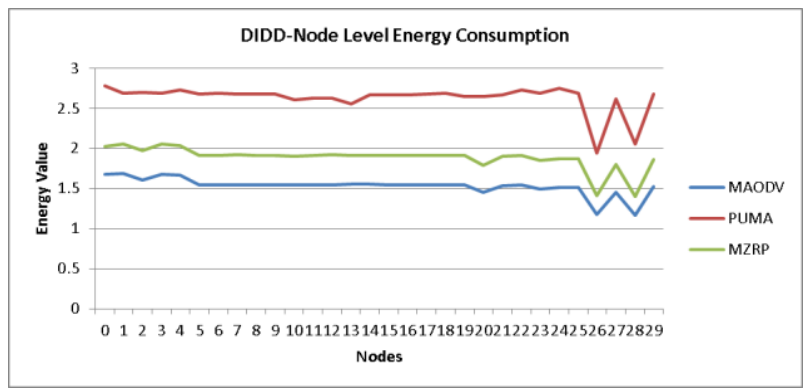

Fig .15 DIDD- Node Level Energy Consumption 


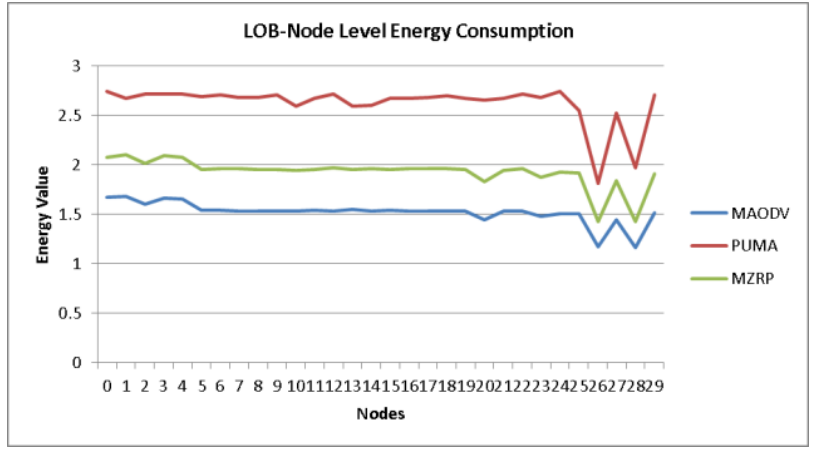

Fig .16 LOB- Node Level Energy Consumption

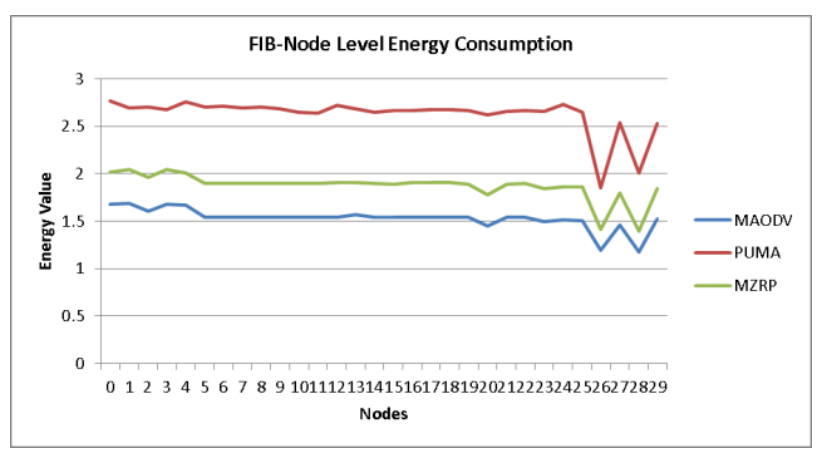

Fig .17 FIB- Node Level Energy Consumption

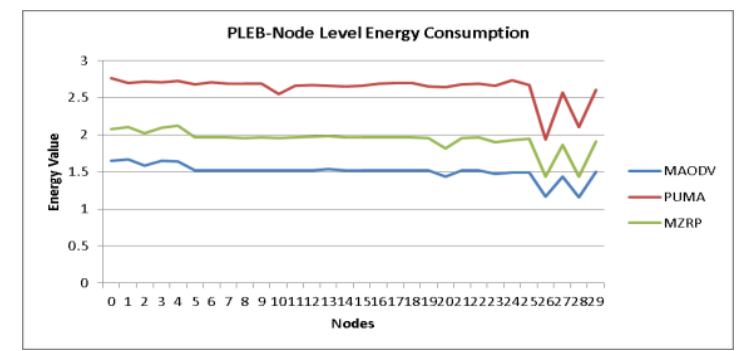

Fig .18 PLEB- Node Level Energy Consumption

It can be observed that MAODV consumed very least amount of energy as compared to MZRP and PUMA. PUMA has the highest energy consumption w.r.t. Backoff algorithms. We can also analyze that node 26 and 28 both consumed less energy w.r.t routing protocols using different backoff algorithms. Now we will examine the node position and behavior in wireless network to identify the reason of variations in node level energy consumption. As per the output of NAM animation, it can be observed that both nodes maintain a specific distance from the group of nodes and did not fully participate in a wireless signal propagation, hence saved their energy levels, but all other nodes were engaged in signal propagation and exhausted their energy at instant level, however energy consumption levels vary using different Backoff algorithms w.r.t routing protocols.

\section{CONCLUSION}

In this paper, we explored the issues and solutions related to resource management for multicast ad hoc networks and performed a simulation based analysis to investigate the impact of MAC layer over the performance of the routing protocols. Simulation results show that selection of Backoff algorithm can enhance the protocol's performance as well as it also affects the resource utilization.

It can be analyzed that Throughput of MAODV is approx. Constant, but in case of PUMA and MZRP, there are some variations in Throughput. MBA and DIDD both are reducing the Throughput of PUMA and it has the lowest value using DBA, MILD and LOB but it is increasing with BEB, EIED, FIB and PLEB. MZRP has the highest Throughput using LOB and it is reduced to its minimum value using DBA. Using BEB, MBA and MILD, It is approx. Constant but in case of EIED and DIDD, it is increasing and with FIB, it is slightly decreasing.

PUMA has the lowest Packet Delivery Ratio as compared to MAODV and MZRP but there are little bit variations in PDR using different Bakoff algorithms w.r.t. routing protocols. MZRP has the highest PDR value using LOB.

PUMA has the highest routing load as compared to MAODV and MZRP with little bit variations. In case of MAODV and MZRP, it remains approx. Constant w.r.t. Backoff algorithms.

MAODV has the lowest End-to-End Delay as compared to MZRP and PUMA. In case of MZRP and PUMA, it is highest using PLEB. There are variations in End-to-End Delay using different Backoff algorithms w.r.t routing protocols.

Energy consumption of MZRP is highest, followed by PUMA and MAODV. There are little bit variations in energy consumption using different Backoff algorithms w.r.t routing protocols.

PUMA has the highest collisions as compared to other protocols w.r.t. Backoff algorithms. MAODV has the minimum number of collisions followed by MZRP with some variations. Using DBA, MILD and FIB, PUMA has the highest number of collisions, using MBA and LOB, number of collisions are decreased to their minimum value. In case of BEB, EIED and PLEB, It is also decreasing.

MAODV has the minimum Average Delay followed by MZRP but it is highest for PUMA with some variations. It is slightly reduced with LOB and PLEB but still remains high as compared to MAODV and MZRP.

PUMA has the highest value of Max. Delay with variation followed by MZRP and MAODV which has the minimum value of Max. Delay. In case of MZRP, it is increasing with LOB and PLEB. Finally, we can conclude that MAODV can manage its performance under the constraint of Backoff algorithms as compared to other protocols. Performance of MZRP little bit suffers from extra control overhead, but it is able to manage energy consumption level. In case of PUMA, due to highest collision ratio, there are variations in the PDR, routing load, delay and energy consumption is also increasing w.r.t. Backoff algorithms.

Simulation results show that there is a need to optimize the resource consumption by managing the behavior of MAC layer as well as the routing layer for multicast communication over ad hoc networks. Network resources can be efficiently utilized by developing a cross layer solution which will be able to reduce the control overhead caused by the dynamic behavior of ad hoc multicast routing protocols/MAC layer.

\section{REFERENCES}

[1] Jean-Marie Vella, Saviour Zammit.A Survey of Multicasting over Wireless Access Networks. IEEE Communications Surveys \& Tutorials.IEEE 2013 June; 15 (2):718-753.

[2] C. Siva Ram Murthy, B.S. Manoj, Ad Hoc Wireless Networks, 14 impression-Pearson, INDIA, 2012.

[3] A. M. A. Mo'men ; Comp. \& Info., Cairo University, 
Egypt ; H. S. Hamza ; I. A. Saroit.A Survey on Security Enhanced Multicast Routing Protocols in Mobile Ad Hoc Networks.HONET- IEEE 7th International Symposium on High-capacity Optical Networks and Enabling Technologies 2010:262-268.

[4] Osamah S. Badarneh andMichel Kadoch.Multicast Routing Protocols inMobile Ad Hoc Networks: A Comparative Survey and Taxonomy.EURASIP Journal on Wireless Communications and Networking.EURASIP-2009:1-42.

[5] Kartik Chawda, Deepmala Gorana.A survey of Energy Efficient Routing Protocol in MANET. IEEE ICECS, 2nd International Conference 2015 Feb:953-957

[6] Y. Sun, R. Sun, F. Jiang, C. Phillips.A new bandwidthefficient multicast routing scheme for mobile Ad hoc Networks. IEEE WTS 2015:1-7.

[7] Ritesh K. Kalle, Maruti Gupta.Aran Bergman, EladLevy,ShantidevMohanty,Muthaiah Venkatachalam, Debabrata Das.Advanced Mechanisms for Sleep Mode Optimization of VoIP Traffic over IEEE $802.16 \mathrm{~m}$. IEEE Globecom 2010:1-6

[8] Jiwen Guo.Secure Minimum-Energy Multicast Tree Based on Trust Mechanism for Cognitive Radio Networks. Springer Wireless Personal Communications 2012 November;67 (2):415-433.

[9] S.M. Lakshmi, K.T. Sikamani.Energy Aware Multicast Opportunistic Routing Protocol (Eamor) To Optimize Lifetime of Manet. IEEE ICCTET 2013 July:210-212.

[10] Di Marco, Stockholm, Fischione, C. ; Athanasiou, G. ; Mekikis, P.-V. MAC-aware routing metrics for low power and lossy networks. IEEE Computer Communications Workshops INFOCOM 2013: 79 - 80

[11] Farooq, M.O.; Inst. of Telematics, Univ. of Luebeck, Luebeck. Germany; Kunz.Proactive Bandwidth Estimation for IEEE 802.15.4-Based Networks . IEEE VTC Spring 2013 June:1-5

[12] Jackson, C.A., Clemson, SC. Russell, H.B. Wolf, B.J, Martin J.Investigation of MAC for a Hierarchical and Heterogeneous Multichannel Ad Hoc Network. IEEE MILCOM 2013 Nov: 188 - 193

[13] Xueyuan Su, Sammy Chan.Bandwidth Allocation in Wireless Ad Hoc Networks:Challenges and Prospects.IEEE Communications Magazine:80-85.
[14] Xiaoying Zhang, Alagan Anpalagan, Lei Guo, Ahmed Shaharyar Khwaja.Energy-Efficient Cooperative MAC Protocol based on Power Control in MANETs. Proc of the 29th International Conference on Advanced Information Networking and Applications IEEE-2015 March:48-53

[15] D. S. Patil, R. Gaikwad.PowerMac: A new protocol design for location based Ad-hoc networks. IEEE ICIC 2015 May:988-911

[16] Xin Zhao,Jun Guo, Chun Tung Chou, Archan Misra, Sanjay K. Jha, "High-Throughput Reliable Multicast in Multi-Hop Wireless Mesh Networks. IEEE Transactions on Mobile Computing 2013 July; 14(4):728 - 741

[17] S. Chettibi, S. Chikhi.Adaptive maximum-lifetime routing in mobile ad-hoc networks using temporal difference reinforcement learning. Springer Evolving Systems.2013 5(2) Auguest:89-108

[18] S. B Kulkarni, Yuvaraju B N.Node connectivity.Energy and Bandwidth Aware Clustering Routing Algorithm for Real-time Traffic Multicasting in MANET. IEEE IACC 2015 June:761-763

[19] Gyanappa A. Walikar, Rajashekar C. Biradar. Energy aware multicast routing in mobile ad-hoc networks using NS-2.IEEE ICECCT. 2015 March:1-7

[20] Yan Sun, Roujia Sun, Fenyu Jiang, Chris Philips.A New Bandwidth -Efficient Multicast Routing Scheme for Mobile Ad hoc networks. IEEE Wireless Telecommunications Symposium (WTS).2015 April:1-7

[21] [21] K. Rajkumar, S. Abinaya, P. Swaminathan. Efficient Resource Allocation in Multicasting over Mobile Adhoc Networks. Indian Journal of Science and Technology, 2014April;7(S4):71-75.

[22] Liao Shengbin and Zhu Xiaoliang.A Survey on Mac Layer in Ad Hoc Wireless Networks.Springer Applied Informatics and Communication, ICAIC 2011;255:691699.

[23] B. C. Kim ; DMC R\&D Center, Samsung Electronics, Suwon, Republic of Korea ; H. Song ; H. S. Lee ; J. S Ma.Performance evaluation of backoff algorithms in multi-hop wireless mesh networks. IEEE 20th International Symposium on Personal, Indoor and Mobile Radio Communications2009 Sept:1347- 1351 